\title{
Young Scientist Prize Announcement for Low Temperature Physics
}

The Commission on Low Temperature Physics (C5) of IUPAP solicits nominations of outstanding young experimental or theoretical physicists for 2022 Young Scientist Prizes. The prize, consists of an IUPAP medal, certificate, and a cash award $(\sim 1000$ Euros), and each winner will give an invited presentation at the 29th International Conference on Low Temperature Physics (LT29), Sapporo, Japan, August 18-24, 2022. Candidates for the prize should have received their $\mathrm{PhD}$ within 8 years from the deadline for submission of nominations. This deadline is December 15, 2021, at 11:59 pm CST.

Nominations should consist of a nomination letter including a suggested citation less than 20 words, two additional letters from experts not in conflict of interest with the candidate, i.e., doctoral, or postdoctoral mentor, or close collaborator, detailing the nominee's qualifications and scientific achievements, a complete $\mathrm{CV}$, and a list of publications in a single pdf file, with the file name being the candidate's name with last name first, e.g., HalperinWP.IUPAP, and mailed to the C5 commission chair, William Halperin: w-halperin@northwestern.edu and secretary Naoto Nagaosa: nagaosa@riken.jp.

IUPAP C5 welcomes applications from women and from underrepresented groups in the field of low temperature physics.

Publisher's Note Springer Nature remains neutral with regard to jurisdictional claims in published maps and institutional affiliations. 